\title{
U.S. Geological Survey Aids Federal Agencies in Obtaining Commercial Satellite and Aerial Imagery
}

\section{Implementation of the Commercial Remote Sensing Space Policy}

The U.S. Geological Survey (USGS) is a leading U.S. Federal civil agency in the implementation of the civil aspects of the Commercial Remote Sensing Space Policy (CRSSP). The USGS is responsible for collecting inter-agency near-term requirements, establishing an operational infrastructure, and supporting the policy and other Federal agencies by:

\section{Collecting Federal civil agency remote sensing data requirements to help support funding strategies and procurement options. The CRSSP Imagery-Derived} Requirements (CIDR) entry tool is designed to collect and provide query and report capabilities on near-term land remote sensing data requirements of U.S. Federal civil agencies. This priority effort is part of the CRSSP implementation and will assist agencies in leveraging resources in areas of common interest. The requirements information gathered and provided will be used to:

- Generate a civil agency requirements database to facilitate partnerships among and within agencies.

- Serve as documented evidence for potential funding initiatives.

- Satisfy user requirements with existing data sources where possible.

- Provide the commercial satellite and aerial industries with a snapshot of civil agency needs; allow industry to respond with accurate and specific data and services.

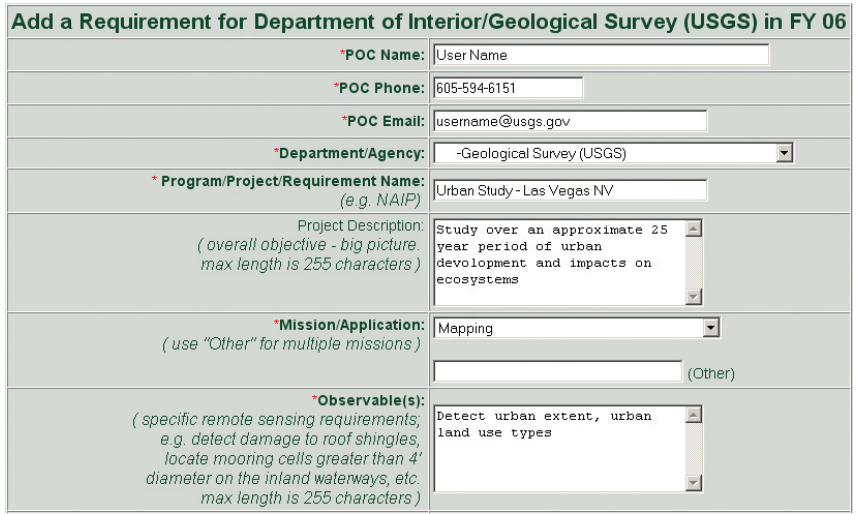

The CIDR tool allows Federal agencies to enter their remote sensing data requirements for upcoming years. This information will be analyzed to find intersections in data requirements and assist users with similar requirements in collaborating on potential purchases. Commercial satellite and aerial vendors may also use the CIDR tool and its information to assist in their data acquisition scheduling for known geographic areas of interest.

Developing an infrastructure to store, manage, and distribute previously acquired commercial remote sensing data. The USGS Commercial Data Purchases (UCDP) Imagery Collection consists of imagery from several commercial vendors. The UCDP Imagery Collection is located at the USGS Center for Earth Resources Observation and Science (EROS) and supports the CRSSP by providing data to qualified users, primarily U.S. Federal agencies, at no cost for File Transfer Protocol (FTP) downloads or at a nominal cost for media. Commercial vendor licensing may restrict access to imagery, and license restrictions may vary for each individual image. However, users have the option to request license upgrades for imagery not currently licensed for their Federal agency. The Commercial Remote Sensing Data Contracts (CRSDC), available through the USGS, handles the license upgrades and enables qualified Federal users to order or download and use a copy of the imagery for a nominal fee.

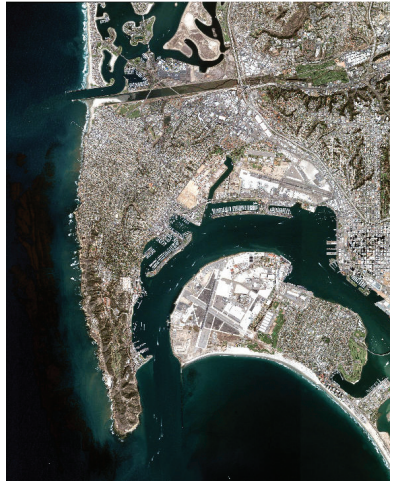

DigitalGlobe: QuickBird-2 03/09/2002, San Diego, CA (Multispectral)

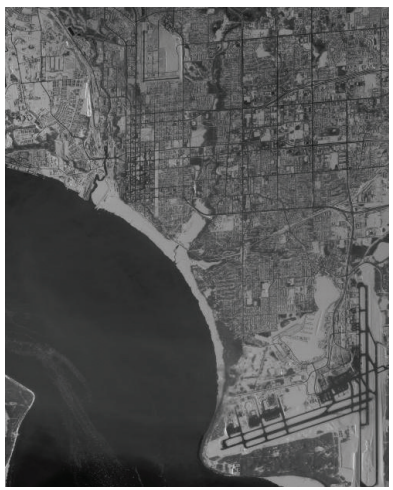

ORBIMAGE: OrbView-3 03/05/2004, Anchorage, AK (Panchromatic)

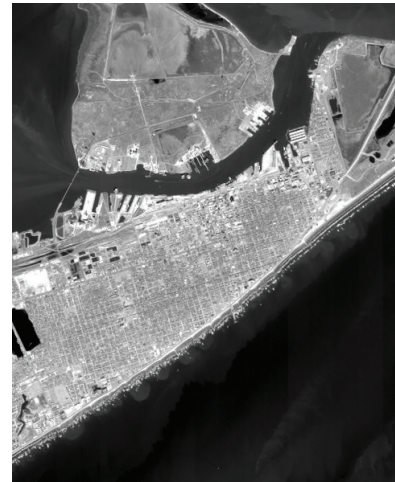

Space Imaging: IKONOS-2 11/11/2003, Galveston, TX (Panchromatic)

Copies of imagery can be ordered or downloaded from http://earthexplorer.usgs.gov.

The only processing parameters available for the imagery are those from the original purchase. (e.g., If an IKONOS image was originally purchased from Space Imaging as a "Standard Ortho" product, UTM projection, WGS84 datum, with GeoTiff format those are the only parameters available.) 
Providing an interface for acquiring commercial remote sensing data from commercial vendors. The USGS Commercial Remote Sensing Data Contracts (CRSDC) provides Federal, State, and local government agencies access to a broad range of commercially available remotely sensed, high-resolution, airborne, and satellite data for both new acquisitions and existing "off-the-shelf" data. These data include orthorectified, panchromatic, natural color, multispectral, infrared, radar, and aerial photography.

The USGS has negotiated advantageous terms on behalf of purchasing organizations, including highly competitive pricing, discounts, and the most flexible and least restrictive licensing arrangements offered to date by the commercial satellite and aerial industries.

These contracts directly support the CRSSP by providing a centralized mechanism for civil agencies to acquire commercial remote sensing products from various commercial vendors.

\section{For more information:}

USGS Commercial Remote Sensing Space Policy (CRSSP)

Home Page

- http://crssp.usgs.gov

CRSSP Imagery-Derived Requirements (CIDR) Entry Tool

- http://cidr.cr.usgs.gov

USGS Commercial Data Purchases (UCDP) Imagery Collection

- http://edc.usgs.gov/products/satellite/ucdp.html

USGS Commercial Remote Sensing Data Contracts (CRSDC)

- http://geodatacontracts.er.usgs.gov/crsdc/index.html

\section{Customer Service:}

U.S. Geological Survey, Center for

Earth Resources Observation and Science

47914 252nd Street

Sioux Falls, SD 57198-0001

Phone: (605) 594-6151

Toll Free: (800) 252-4547

TDD: (605) 594-6933

Fax: (605) 594-6589

Email:_crssp@usgs.gov

Business hours: Monday through Friday, 8:00 a.m. to 4:00 p.m. central time 